\title{
Revealing Cosmic-Ray acceleration in the SNR W51C
}

\section{Tobias Jogler*}

SLAC National Accelerator Laboratory, 2575 Sand Hill Road M/S 29, Menlo Park, CA 94025, USA

Erlangen Centre for Astroparticle Physics, D-91058 Erlangen, Germany

E-mail: joglereslac.stanford.edu

\section{on behalf of the Fermi-LAT collaboration}

\begin{abstract}
SNR are commonly assumed to accelerate the cosmic rays of $\mathrm{E}<1 \mathrm{PeV}$ observed at Earth. SNRs that interact with molecular clouds (MCs) are very promising targets to distinguish between leptonic and hadronic-induced gamma-ray emission. One of the brightest Fermi/LAT-detected SNRs interacting with a MC is W51C. Here we present a very detailed analysis of 5 years of Fermi/LAT data revealing a very significant low-energy break in the gamma-ray spectrum associated with the production threshold of neutral pions. This unmistakable feature of CR-acceleration in W51C is further investigated and we present a detailed modelling of the source with various gamma-ray production mechanisms. We finally compare the derived properties of W51C with those of the other known CR accelerators W44 and IC443. The here presented results will be soon published in a dedicated paper containing all the details.
\end{abstract}

The 34th International Cosmic Ray Conference,

30 July- 6 August, 2015

The Hague, The Netherlands

${ }^{*}$ Speaker. 